\title{
Survey of US Patients with Multiple Sclerosis: Comparison of the New Electronic Interferon Beta-1b Autoinjector (BETACONNECT ${ }^{\mathrm{TM}}$ ) With Mechanical Autoinjectors
}

\author{
Donald A. Barone · Barry A. Singer · Lubo Merkov • Mark Rametta • \\ Gustavo Suarez
}

Received: March 17, 2016 / Published online: June 8, 2016

(c) The Author(s) 2016. This article is published with open access at Springerlink.com

\section{ABSTRACT}

Introduction: Patients with multiple sclerosis (MS) generally undergo long-term treatment with disease-modifying therapies (DMTs). In the US, patients taking glatiramer acetate, interferon beta- $1 \mathrm{a}$, or interferon beta- $1 \mathrm{~b}$, typically use a mechanical autoinjector. Recent survey results have shown that using an electronic autoinjector, such as BETACONNECT $^{\mathrm{TM}}$ (Bayer Pharma AG) for interferon beta-1b/Betaseron ${ }^{\circledR}$ (Bayer Pharma AG) may reduce injection discomfort and

Enhanced content To view enhanced content for this article go to http://www.medengine.com/Redeem/B7D 4F0602A357686.

Electronic supplementary material The online version of this article (doi:10.1007/s40120-016-0047-3) contains supplementary material, which is available to authorized users.

D. A. Barone $(\varangle)$

School of Osteopathic Medicine, Rowan University, Stratford, NJ, USA

e-mail: baroned1@rowan.edu

B. A. Singer

Missouri Baptist Medical Center, St Louis, MO, USA

L. Merkov · M. Rametta · G. Suarez

Bayer HealthCare Pharmaceuticals, Whippany, NJ, USA increase patient satisfaction with treatment. The aim of the current survey was to assess patient perceptions of BETACONNECT compared with mechanical autoinjectors using a survey integrated with demonstrations and simulated injections with BETACONNECT.

Methods: Patients with MS currently using mechanical autoinjectors for glatiramer acetate/Copaxone $^{\circledR} \quad$ (Teva Pharmaceuticals USA, Inc.), interferon beta-1a/Rebif ${ }^{\circledR}$ (EMD Serono Inc.), or interferon beta-1b/Extavia ${ }^{\circledR}$ (Novartis Pharmaceuticals Corp.), participated in a 60-min in-person interview. Patients rated the importance of 18 ideal autoinjector attributes, and the performance of their current autoinjectors across these attributes. BETACONNECT was demonstrated and patients performed simulated injections with BETACONNECT before rating it across the same attributes. Patient overall autoinjector preference was assessed.

Results: Ninety patients completed the survey: 63 were using autoinjectors for Copaxone, 25 for Rebif, and 2 for Extavia. BETACONNECT scored higher than mechanical autoinjectors across all 18 attributes. The top attributes of an ideal autoinjector were the injection process is 
easy overall, easy to push the button to start the injection, and autoinjector is comfortable to hold during injections. Unique BETACONNECT features most valued by patients were the built-in dwell time, self-check function, greater ability to customize injections, adjustment of injection speed, low injection noise, and automatic needle retraction. Overall, 75 out of 90 patients (83\%) expressed a preference for BETACONNECT over their current autoinjector. Conclusion: BETACONNECT attributes and features were highly rated by patients, compared with both an ideal autoinjector and their current mechanical autoinjectors. These findings suggest that the use of BETACONNECT may increase patient satisfaction and potentially increase overall medication adherence.

Funding: Bayer HealthCare Pharmaceuticals.

Keywords: Autoinjector; BETACONNECT; Disease-modifying therapies; Patient survey; Preference; Relapsing-remitting multiple sclerosis

\section{INTRODUCTION}

Multiple sclerosis (MS) is a chronic autoimmune disease that can affect all parts of the central nervous system. Most patients experience periods of neurological relapses and remissions early in their disease course, followed by progressive neurological deterioration [1]. There are no curative treatments for MS, but disease-modifying therapies (DMTs) such as interferon beta- $1 \mathrm{~b}$ are considered effective therapies for the management of MS [2]. Long-term management with DMTs can alter the pathological immune responses associated with MS, prevent relapses, and reduce the risk of disease progression [3]. Optimal management of
MS with DMTs depends on a number of factors, including starting the treatment early and adherence to the medication regimen [2, 4]. Nevertheless, poor adherence to DMTs is frequently seen among patients with MS [4] and can lead to increased risk of relapse and higher medical costs $[5,6]$.

Several DMTs are given as patient-administered injections via the use of an autoinjector, as these devices can reduce patients' anxiety about self-administered injections and contribute to a lower rate of injection site reactions [7-9]. Moreover, autoinjector use may be associated with improved adherence to injectable therapy [10]. Innovations in autoinjector design have advanced the management of MS using injectable DMTs. For example, electronic autoinjectors can improve the overall injection experience by employing new technology. Some of the new features of electronic autoinjectors include silent injections owing to a motorized injection process, hidden needles, individual injection settings (e.g., adjustable injection speed and depth), skin sensors to detect that the device is positioned correctly before injection, recording of dose history, and injection reminder functions $[4,7,11]$.

The new fully electronic BETACONNECT ${ }^{\mathrm{TM}}$ autoinjector (Bayer Pharma AG) tested in the current survey was developed to provide subcutaneous interferon beta- $1 \mathrm{~b}$ therapy (Betaseron ${ }^{\circledR}$; Bayer Pharma AG) while incorporating innovative features to optimize patients' injection experience. These include advances in autoinjector design and technology as outlined in the following section, which may enhance overall patient comfort and satisfaction, improving the management of MS. It is clear that patients' perceptions and preferences are vitally important, as when taken 
into account they can increase treatment effectiveness and reduce healthcare costs [12].

The objective of the current survey was to use a comprehensive survey-based approach to assess patient perceptions of BETACONNECT in comparison with their experiences using mechanical autoinjectors that are available in the US. This survey builds upon results from recently published surveys which have shown high levels of satisfaction with BETACONNECT and preference over previous methods for injecting interferon beta-1b therapy [13, 14]. The current survey is the first to compare BETACONNECT with mechanical autoinjectors.

\section{METHODS}

\section{Autoinjectors}

Figure 1 shows the BETACONNECT autoinjector. Note that the Rebismart $^{\circledR}$ autoinjector (Merck Serono) was not included as it has not been approved for use in the US, and we also excluded those using the Rebidose ${ }^{\circledR}$ 'pen' device (EMD Serono Inc.) as this is not an

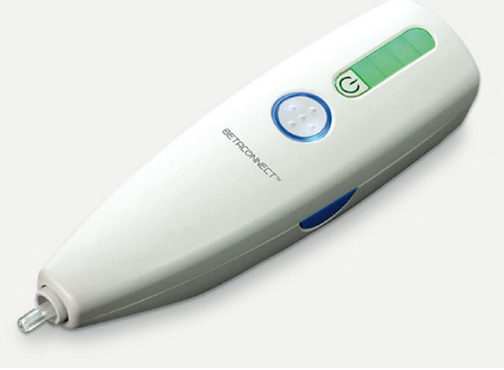

Fig. 1 The BETACONNECT autoinjector. Comparator autoinjectors images and details are available from the following websites: Copaxone Autoject/Autoject 2 [23], Extavia Auto-injector/Auto-injector II [24], Rebif Rebiject/Rebiject II [25]. Note: the RebiSmart autoinjector was not included as it has not been approved for use in the US. Pen injector devices such as the Rebif Rebidose were also excluded as they are not autoinjectors autoinjector. Patients using injectable DMTs such as interferon beta-1a, whether as Avonex $^{\circledR}$ (Biogen) or Plegridy ${ }^{\circledR}$ (Biogen) formulations, were also excluded from this survey as they too use pen devices rather than autoinjectors.

A BETACONNECT device was demonstrated to and used by survey participants to perform a simulated injection as part of this market research. Its main features include an intuitive user interface and an ergonomic design making it potentially easier to handle and allowing one-handed injections. It has adjustable injection speed and depth to allow customized injection settings, and a low-force safety release to ensure the device is positioned correctly at the time of the injection. The nearly silent electronic injection features four-phase injection technology: (1) automatic needle insertion, (2) delivery of the medication, (3) dwell time, when the needle remains in the skin momentarily, and, (4) automatic needle retraction. The autoinjector provides visual and audible signals that the injection has finished. It also features a dose reminder function and audible and visual indicators of battery status. Finally, the device automatically records data such as injection date and time, injection depth, injection speed, and injection volume. In due course, BETACONNECT will offer the possibility to share data with healthcare providers via an app called myBETAapp $^{\circledR}$ and the Navigator, a dashboard monitoring application, to enhance communication between patients and healthcare professionals (myBETAapp and Navigator are currently under FDA review).

\section{Participants}

Recruitment of patients for this survey was organized through an independent external 
market research company that conducted screening telephone calls to select patients with MS from their database of participants who had previously expressed a willingness to participate in market research projects. Patients were included if they lived in the US, were aged 18-70 years, had a relapsing form of MS, and sometimes or always used an autoinjector to self-administer either glatiramer acetate/ Copaxone $^{\circledR}$ (Teva Pharmaceuticals USA, Inc.; Copaxone Autoject or Autoject 2), interferon beta-1b/Extavia ${ }^{\circledR} \quad$ (Novartis Pharmaceuticals Corp.; Extavia Auto-Injector or Auto-Injector II), or interferon beta-1a/Rebif ${ }^{\circledR}$ (EMD Serono Inc.; Rebif Rebiject or Rebiject II). Exclusion criteria were a diagnosis less than 6 months ago or a diagnosis of primary progressive MS (PPMS).

\section{Survey Instruments and Data Collection}

Data were collected from patients with MS between August and September 2015 through in-person 60-min structured interviews in 12 cities throughout the US: Philadelphia, New York City, Atlanta, Miami, Tampa, Chicago, Las Vegas, Phoenix, Houston, Dallas, Los Angeles and Seattle. Signed informed consent to participate was obtained from all participants, who also had to bring their current autoinjector with them.

The interview consisted of four stages. Stage 1: introduction and background. In this stage patients gave information on their MS diagnosis, and their MS disease and treatment history. Stage 2: unmet needs and satisfaction with current autoinjector. In this stage patients were presented with a list of 18 possible autoinjector attributes (Table S1 in the supplementary material) and asked to indicate how important each attribute would be in an ideal autoinjector, rating them from 1 (not important) to 10 (most important) and instructed to only assign the maximum rating of 10 to one attribute. Patients indicated how well their own autoinjectors performed across the same 18 attributes, rating from 1 (not well at all) to 10 (very well). Stage 3: BETACONNECT demonstration and "simulated injection". Here, the interviewer showed the patients a BETACONNECT autoinjector with all branding and identifiers masked. For the purposes of this research, the BETACONNECT autoinjector contained an empty syringe, but no needle. The patients were first shown how to use BETACONNECT and then how to perform a "simulated injection". The patients next used BETACONNECT, first being guided through the device features and the injection process and then performing simulated self-injections at various injection sites. Stage 4: BETACONNECT assessment. Here, patients were again presented with the list of 18 autoinjector attributes (Table S1) and asked to rate how well the BETACONNECT autoinjector performed across these attributes, rating from 1 (not well at all) to 10 (very well). Patients compared their current autoinjector with BETACONNECT, agreeing or disagreeing with a number of statements by assigning a rating from 1 (strongly disagree) to 10 (strongly agree; Table S2 in the supplementary material). Finally, patients answered general queries about their choices, including which features they liked the most and the least, and why, and whether they would prefer using BETACONNECT or their autoinjector. Specifically, patients were asked "Assuming your current MS medication can use the new autoinjector, please select the one statement that best describes your overall preference: (1) I would rather use the new autoinjector than my current autoinjector or (2) I would rather continue to use my current autoinjector than this new autoinjector." 
The survey was funded by Bayer HealthCare Pharmaceuticals, Inc., Whippany, New Jersey, USA. Patients were compensated for their time and travel costs to the market research facilities. All procedures followed were in accordance with the 1964 Declaration of Helsinki and its subsequent revisions. This article describes a non-interventional survey, and so does not involve any new studies of human or animal subjects performed by any of the authors.

\section{RESULTS}

\section{Demographic Characteristics}

A total of 90 respondents with MS agreed to participate, and completed the survey (Table 1). Of these, 63 (70\%) were using autoinjectors for Copaxone, 25 (28\%) for Rebif, and 2 (2\%) for Extavia. This distribution of patients is somewhat representative of these products' current sales market share for injectable DMTs within the US. Because of the small number of patients taking Extavia, no individual comparisons were made for this group with BETACONNECT but the results for the Extavia group were included in overall (all patient) comparisons. Approximately three-quarters $(78 \%)$ of respondents were female.

\section{Ideal Autoinjector Attributes}

Figure 2 shows the most important features of an ideal autoinjector according to the percentage of patients who assigned a high score of 8,9 , or 10 (top three scores on a 10-point scale) for each attribute. The most highly valued attributes were: the injection process is easy overall (96\%), easy to push the button to start the injection (88\%), and autoinjector is comfortable to hold during injections (86\%). Other features that were rated highly included the ability to use the autoinjector without help from others (83\%), adjustable injection depth (81\%), and being able to reach different injection sites (78\%). Patients were also asked to assign the maximum rating of 10 to only one of the attributes. The ideal autoinjector attribute given the most number of maximum scores was the injection process is easy overall (26\%), with the ability to use the autoinjector without help from others and the sound of injection is minimal (quiet) both gaining the next highest number of maximum scores (11\% each).

\section{Comparison of Current Autoinjector With BETACONNECT}

Patients rated their own autoinjector using the same ideal autoinjector attributes mentioned previously, and results for individual autoinjectors are displayed in Fig. 3. This figure also shows the results of patients rating BETACONNECT across the same attributes following a demonstration and simulated self-injections with BETACONNECT. Moreover, this figure shows that BETACONNECT outperforms the Copaxone Autoject or Autoject 2 and Rebif Rebiject or Rebiject II across all the attributes. Figure 4 shows the percentage of patients who assigned a high score of 8,9 , or 10 on a 10-point scale to statements concerning their attitudes and perceptions towards BETACONNECT in comparison with their current autoinjector. Built-in dwell time (i.e., the time the needle remains in the skin after the medication has been injected) was most valued (97\%), followed by the self-check feature (84\%), greater ability to customize injections (84\%) and adjustment of injection speed (83\%). The subsequent assessment of overall autoinjector preference 
Table 1 Demographic characteristics of survey participants $(n=90)$

\begin{tabular}{lc}
\hline Characteristic & Number (\%) \\
\hline Females & $70(78 \%)$ \\
Age (years) & $3(3 \%)$ \\
$18-30$ & $16(18 \%)$ \\
$31-40$ & $25(28 \%)$ \\
$41-50$ & $42(47 \%)$ \\
$51-65$ & $4(4 \%)$ \\
$66-70$ & \\
Geographic distribution & $16(18 \%)$ \\
Northeast (Philadelphia, New York City) & $22(24 \%)$ \\
Southeast (Atlanta, Miami, Tampa) & $7(8 \%)$ \\
Midwest (Chicago) & $27(30 \%)$ \\
Southwest (Las Vegas, Phoenix, Houston, Dallas) & $18(20 \%)$ \\
West (Los Angeles, Seattle) & \\
Current MS medication ${ }^{a}$ and autoinjector ${ }^{b}$ & $63(70 \%)$ \\
Copaxone (Copaxone Autoject/Copaxone Autoject 2) & $25(28 \%)$ \\
Rebif (Rebif Rebiject/Rebif Rebiject II) & $2(2 \%)$ \\
Extavia (Extavia Auto-injector/Extavia Auto-injector II) & \\
MS type & $87(97 \%)$ \\
Relapsing-remitting MS (RRMS) & $3(3 \%)^{c}$ \\
Secondary progressive MS (SPMS) & $1(1 \%)$ \\
Clinically isolated syndrome (CIS) & $0(0 \%)$ \\
\hline
\end{tabular}

$M S$ Multiple sclerosis

${ }^{a}$ The distribution of patients taking these medications is somewhat representative of these drugs market share within the US, hence the small proportion taking Extavia

b It was not possible for this study to include the RebiSmart autoinjector as it is not licensed in the US where this survey took place. Pen injector devices such as the Rebif Rebidose were also excluded as they are not autoinjectors

${ }^{c}$ Includes one patient with a diagnosis of secondary progressive MS and relapsing-remitting MS

revealed that $83 \%$ of patients preferred built-in dwell time and automatic needle BETACONNECT to their current autoinjector, as they agreed with the statement "I would rather use the new autoinjector than my current autoinjector" (Fig. 5). The main reasons for the preference for BETACONNECT were ease of injection, user-friendly functions such as retraction, and quiet injections. Preference was also analyzed by patient age group (Table 2), showing a high (100\%) preference for BETACONNECT in younger patients (i.e., 18-40 years of age), with older patients slightly less likely to switch. 


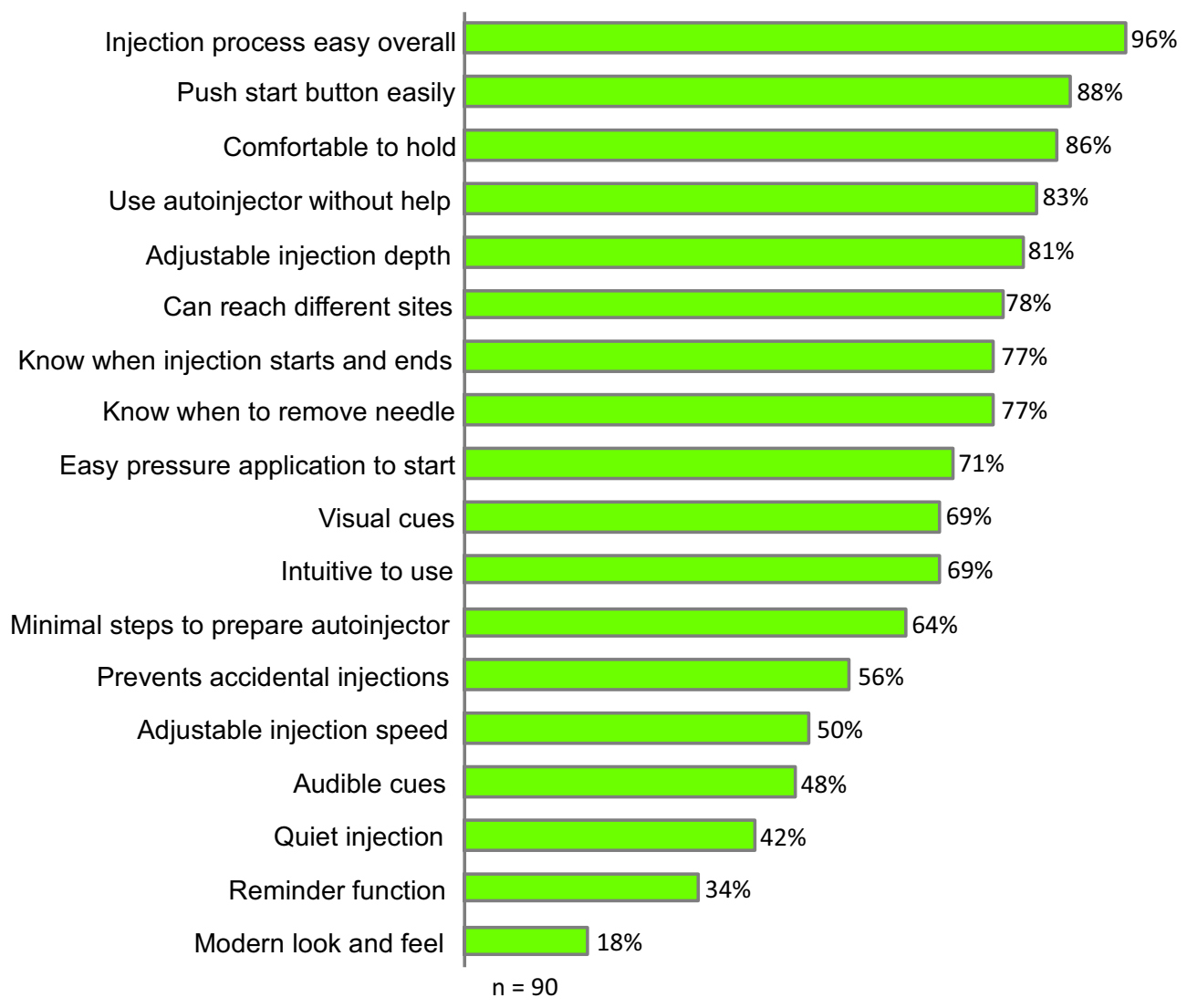

Fig. 2 Ideal autoinjector attributes rating from 1 to 10 (where 10 is most important and 1 least important). The percentages represent the proportion of patients who selected a rating of 8,9 or 10 for each attribute

\section{DISCUSSION}

The first part of this survey investigated what patients thought might be ideal autoinjector attributes. The most important features focused on being easy to use, comfortable to hold, customization for optimal comfort during injections, and being able to self-inject without help and so maintain independence. These results are in broad agreement with previous published results. For example, a survey of patients with MS showed that the main reason for not self-injecting was physical difficulty in performing the injections (57\%) [15]. Another survey showed that major concerns preventing self-injection were fear of performing injections properly and ability to access various injection sites (both 47\%) [16]. A decline in fine motor skills is a common problem among patients with MS, reducing their ability to administer injections [4]. Thus, improvements in autoinjector ease of use are vital to allow patients to fulfill their desire to maintain their independence by performing self-injections.

It is interesting to note that while some of the features of BETACONNECT were not thought to be of major importance among the ideal autoinjector attributes (e.g., adjustable injection speed), they were rated more highly once patients had performed simulated injections with BETACONNECT. This may be because patients were not aware what features were potentially feasible as they 


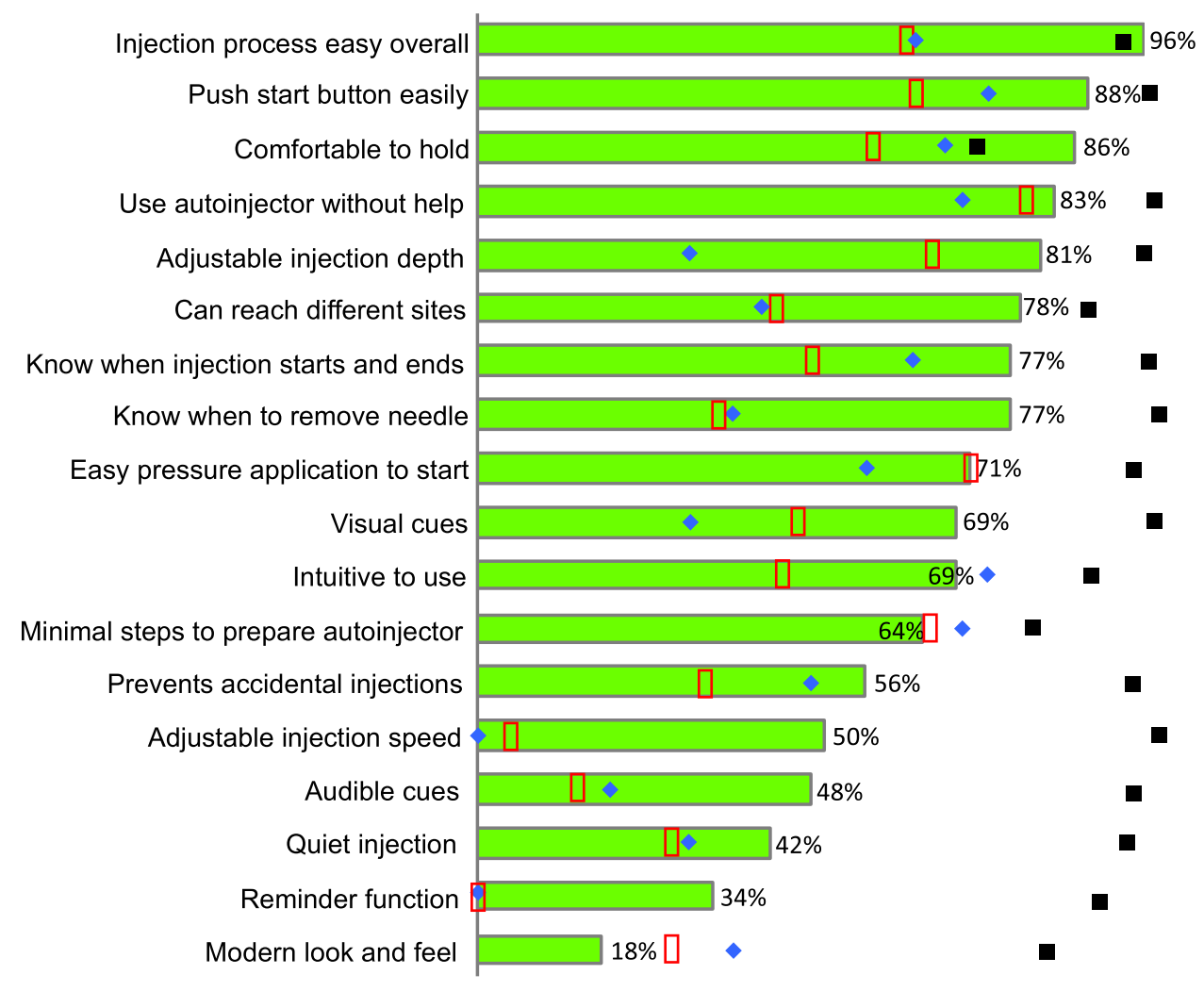

- BETACONNECT $(n=90)$ - Rebiject/Rebiject II $(n=25)$

(1) Autoject/Autoject 2 ( $n=63)$

Fig. 3 BETACONNECT, Autoject and Rebiject, compared individually against an ideal autoinjector across 18 attributes. Attribute ratings for an ideal autoinjector are also shown (green bars). Autoject, Rebiject and BETACONNECT were rated based on how well they performed across the attributes, from 1 (not well) to 10 (very well). For each attribute, the percentage of patients who selected a rating of 8,9 , or 10 for each autoinjector using symbols as

had not encountered them at this stage of the survey. Another example of this phenomenon was quietness of injection, which was overall rated rather low among the initial assessment of ideal autoinjector attributes as assessed by the percentage giving a score of 8,9 , or 10 , even though it was the second most desirable attribute according to the number of maximum scores. The results reveal an interesting dichotomy: prior to encountering BETACONNECT patients thought quiet injections were either not an important shown in the legend. Note: we did not compare Extavia patients separately vs. other groups because there were only two patients using Extavia. The other groups had enough patients for us to analyze and compare them. The two patients using Extavia autoinjectors were included in the total number of 90 patients (all patients following the demonstration and simulated injections with BETACONNECT)

attribute or regarded it as major issue. After patients had experienced quiet simulated injections using BETACONNECT many changed their opinions, as $63 \%$ of patients thought that lack of a loud noise upon injection would reduce their anxiety (Fig. 4).

While BETACONNECT outperformed Copaxone Autoject or Autoject 2 and Rebif Rebiject or Rebiject II over all of the autoinjector attributes, this outperformance was particularly marked in features that were not highly rated in the initial assessment of 


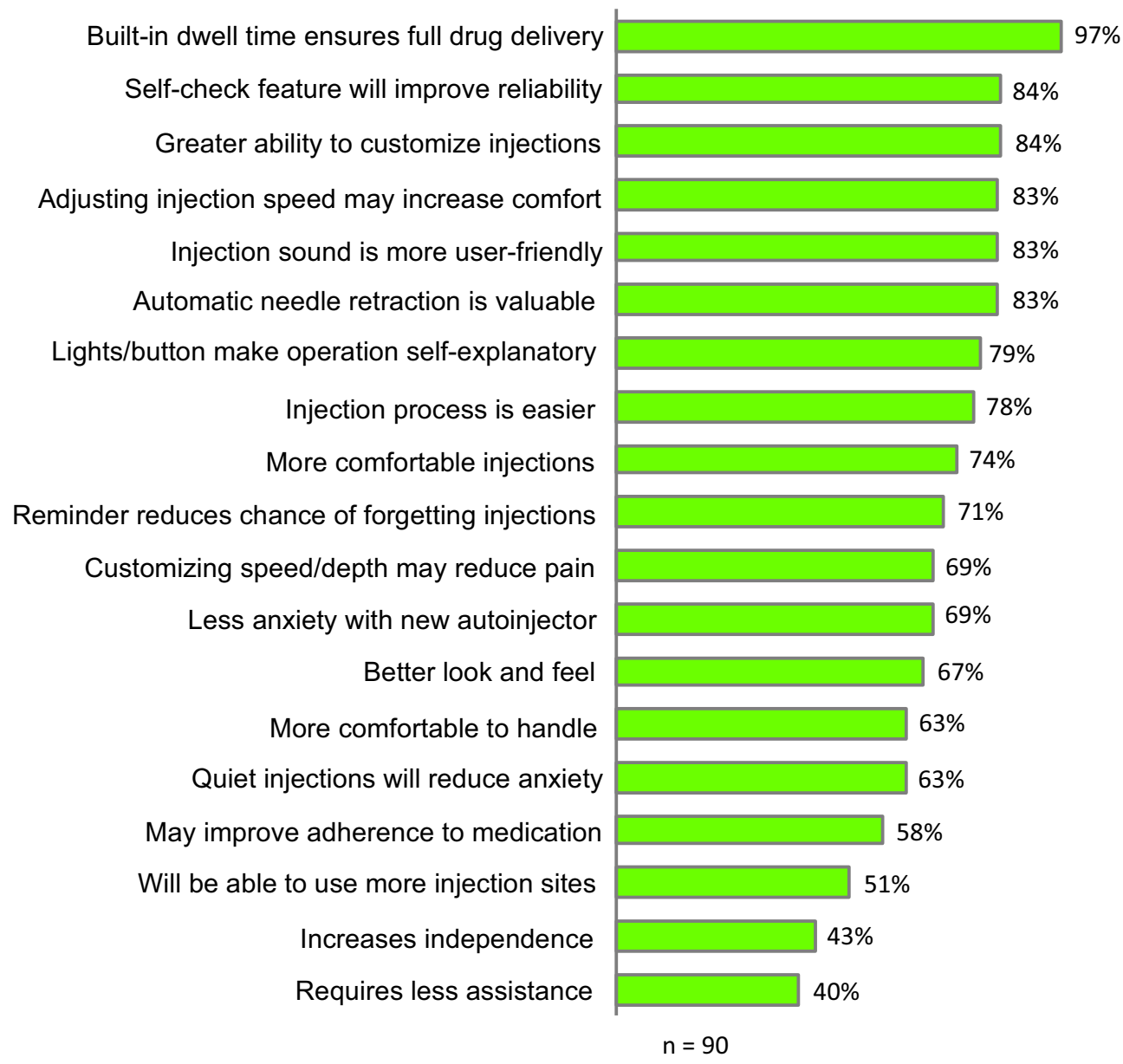

Fig. 4 Attitudes and perceptions towards BETACONNECT in comparison with patients' current autoinjectors, showing the percentage who scored statements 8,9 , or 10

attributes prior to performing simulated injections with the new autoinjector (e.g., injection reminders, audible cues, quietness of injection, ability to adjust injection speed, and knowing when to remove the needle). This strongly suggests that the improvements in autoinjector design featured in BETACONNECT may be meeting the patients' needs, even needs that they were unaware of before using it. It is worth noting here that we were unable to include the RebiSmart autoinjector in the survey as it has not been approved for use in the US. (where 1 is strongly disagree and 10 is strongly agree). The percentages represent the proportion of patients who selected a rating of 8,9 , or 10 for each statement

The main reasons for the preference for BETACONNECT in the current survey appear to be the ease of injection (e.g., minimal pressure needed to press the injection button, helping patients to reach various injection sites across the body); user-friendly features such as built-in dwell time and automatic needle retraction; visible/audible cues denoting when the injection is complete to prevent premature needle withdrawal; and the quiet nature of the injection. Likewise, the minority who preferred to use their current autoinjector tended to be older, more concerned about potential 
I would rather continue to use my current autoinjector than this new autoinjector
I would rather use the new autoinjector

than my current autoinjector

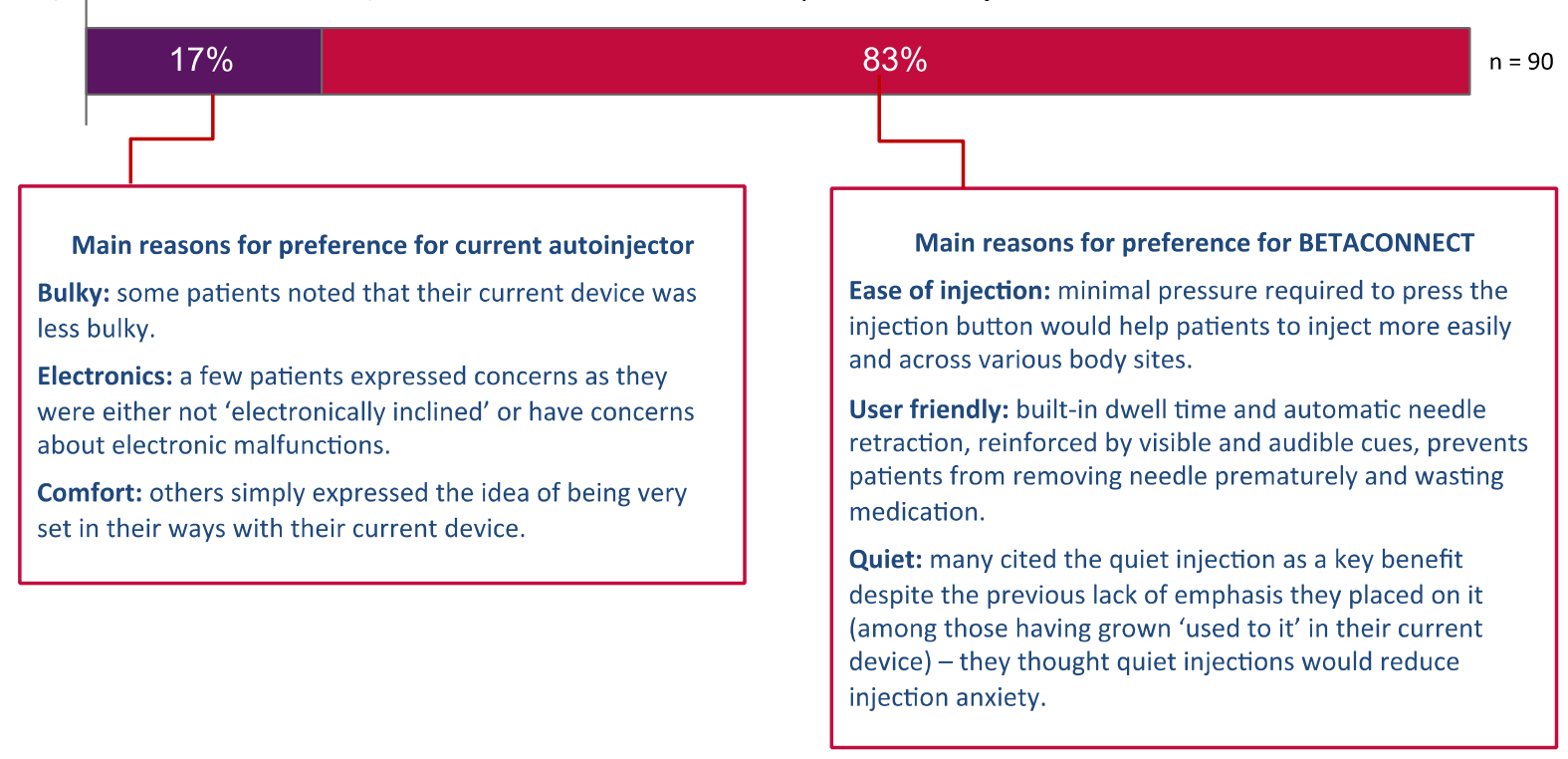

Fig. 5 Patient overall autoinjector preference and main underlying reasons

Table 2 Preference for BETACONNECT by age group

\begin{tabular}{lc}
\hline Age (years) & $\begin{array}{l}n^{\mathbf{a}} / \boldsymbol{N}^{\mathbf{b}}(\%) \text { preferring } \\
\text { BETACONNECT }\end{array}$ \\
\hline $18-30$ & $3 / 3(100 \%)$ \\
$31-40$ & $16 / 16(100 \%)$ \\
$41-50$ & $22 / 25(88 \%)$ \\
$51-65$ & $31 / 42(74 \%)$ \\
$66-70$ & $3 / 4(75 \%)$ \\
\hline
\end{tabular}

${ }^{a}$ Number of patients in each age group who would rather use BETACONNECT than their current autoinjector

b Total number of patients in each age group

electronic malfunctions, preferred their 'less bulky' current device, or were just used to their current autoinjector and unwilling to change.

The BETACONNECT attributes valued by most patients were knowing when to pull the needle out after an injection (99\%), adjustable injection speed (99\%), visual cues to show injection progress (98\%), being able to use without help (98\%), easy to push a button to start injections (97\%), and being easy to tell when an injection starts and stops (97\%; Fig. 3). In addition, the new autoinjector features valued by patients over their current auto injector were the built-in dwell time (97\%), self-check function (84\%), injection speed adjustment (83\%), low injection noise (83\%), and automatic needle retraction (83\%; Fig. 4). Considering these results together, they reflect the key features of BETACONNECT that make it particularly useful for patients with MS, notably automatic needle insertion and retraction, audio/visual cues that indicate the injection progress and the successful end of an injection, and adjustable injection speed and depth to allow customized injection settings. Automatic needle insertion, dwell time, and retraction, in conjunction with visual and audible signals, ensure that injections are delivered completely. This meets common concerns that the injection might not be performed correctly [16]; many patients in the current survey said they counted to ten when using their mechanical 
autoinjector to avoid spilling medication. Moreover, the customized settings of BETACONNECT may help overcome a major barrier to DMT adherence for patients with MS, as one survey showed that $34 \%$ of patients did not use their injection device as they wanted to control their injections, including control of the speed and depth of needle insertion [16]. BETACONNECT also features a unique injection reminder function. While only $71 \%$ of patients thought that the reminder function might be helpful (Fig. 4), this feature may become more important with use, as forgetting to take medications for MS is the most common reason for non-adherence to MS drug therapy, owing to the cognitive changes that form part of the natural disease course $[17,18]$.

Strengths of the survey included the in-depth nature of each interview, lasting about an hour for each patient, as well as the time spent by patients handling and performing simulated injections with BETACONNECT. Potential weaknesses of the current survey are that it was performed in an uncontrolled setting and without a longitudinal follow-up, and that it had a relatively small sample size. Paying patients' expenses to participate in the survey might bias them towards BETACONNECT, but patients were also blinded to any name or branding on the BETACONNECT to help reduce such prejudice. Another potential criticism of the current survey is the relative distribution of autoinjector types, with $70 \%$ using autoinjectors for Copaxone, 28\% for Rebif, and only $2 \%$ for Extavia. However, this distribution reflects the generally higher prevalence of Copaxone and Rebif patients in the US and the difficulty in recruiting patients taking Extavia, given the low level of Extavia use. In addition, from January to December 2015, only $29 \%$ of total Rebif prescriptions were for Rebif Rebidose and $71 \%$ were for Rebif that could be injected with a mechanical autoinjector [19]. Betaseron patients were excluded as patient satisfaction surveys with patients using BETACONNECT were conducted previously $[13,14]$. The current survey does not contain adherence data, so we cannot know for sure that BETACONNECT will increase adherence to DMTs. Clearly, adherence to DMTs is of great importance as it may reduce the incidence of relapses, lead to fewer hospitalizations and avoid excess use of healthcare resources [4]. Other studies have shown promising adherence rates with other electronic autoinjectors used by patients with MS $[20,21]$. Several BETACONNECT studies to investigate adherence are on-going or are planned to start shortly to investigate this issue.

\section{CONCLUSIONS}

Adherence to DMTs is vital to optimize treatment efficacy and so improve patient outcomes, but it nevertheless remains a challenge [4, 22]. Ongoing improvements in autoinjector design and functionality as exemplified by BETACONNECT may allow patients with MS to overcome many self-injection difficulties that can, for example, result in poor adherence to MS drug therapy. In the current survey, BETACONNECT attributes and features were rated highly by patients, compared with both an ideal autoinjector and their current mechanical autoinjector. As such, these findings suggest that the use of BETACONNECT may increase patient satisfaction and potentially increase overall medication adherence.

\section{ACKNOWLEDGMENTS}

All named authors meet the International Committee of Medical Journal Editors (ICMJE) 
criteria for authorship for this manuscript, take responsibility for the integrity of the work as a whole, and have given final approval to the version to be published. Valuable consultation was provided throughout the project by $\mathrm{Dr}$ Martina Sintzel (mcs medical communication services, Küsnacht, Switzerland) and was funded by Bayer HealthCare Pharmaceuticals. We are also grateful for medical writing services provided by Dr Richard Clark (freelance medical writer, Dunchurch, Warwickshire, UK) which were funded by Bayer HealthCare Pharmaceuticals. BluePrint Research Group was employed as an independent external market research company and market research facilities were provided by Schlesinger Associates. Financial support for this project was provided by Bayer HealthCare Pharmaceuticals.

Disclosures. Donald Barone has acted as a speaker and/or consultant for Acorda, Bayer, Biogen, EMD Serono, Sanofi/Genzyme, Teva and has received research support from Acorda, Bayer, Biogen, and EMD Serono. Barry Singer has acted as a speaker and/or consult for Acorda, Bayer, Biogen, EMD Serono, Genentech, Novartis, Pfizer, Sanofi Genzyme, Teva and has received research funding from Acorda, Biogen, Sanofi Genzyme, Novartis, and Roche. Lubo Merkov, Mark Rametta, and Gustavo Suarez are employees of Bayer HealthCare Pharmaceuticals, Whippany, NJ, USA.

Compliance with Ethics Guidelines. All procedures followed were in accordance with the 1964 Declaration of Helsinki and its subsequent revisions. Informed consent was obtained from all patients included in the survey. This article describes a non-interventional survey, and so does not involve any new studies of human or animal subjects performed by any of the authors.

Open Access. This article is distributed under the terms of the Creative Commons Attribution-NonCommercial 4.0 International License (http://creativecommons.org/licenses/ by-nc/4.0/), which permits any noncommercial use, distribution, and reproduction in any medium, provided you give appropriate credit to the original author(s) and the source, provide a link to the Creative Commons license, and indicate if changes were made.

\section{REFERENCES}

1. Compston A, Coles A. Multiple sclerosis. Lancet. 2008;372:1502-17.

2. Gold R, Wolinsky JS, Amato MP, Comi G. Evolving expectations around early management of multiple sclerosis. Ther Adv Neurol Disord. 2010;3(6):351-67.

3. Ziemssen T, De Stefano N, Pia Sormani M, Van Wijmeersch B, Wiendl H, Kieseier BC. Optimizing therapy early in multiple sclerosis: an evidence-based view. Mult Scler Relat Disord. 2015;4(5):460-9.

4. Lugaresi A, Rottoli MR, Patti F. Fostering adherence to injectable disease-modifying therapies in multiple sclerosis. Expert Rev Neurother. 2014;14(9):1029-42.

5. Tan H, Cai Q, Agarwal S, Stephenson JJ, Kamat S. Impact of adherence to disease-modifying therapies on clinical and economic outcomes among patients with multiple sclerosis. Adv Ther. 2011;28(1):51-61.

6. Cohen BA, Coyle PK, Leist T, Oleen-Burkey MA, Schwartz M, Zwibel H. Therapy optimization in multiple sclerosis: a cohort study of therapy adherence and risk of relapse. Mult Scler Relat Disord. 2015;4(1):75-82.

7. Lugaresi A. Addressing the need for increased adherence to multiple sclerosis therapy: can delivery technology enhance patient motivation? Expert Opin Drug Deliv. 2009;6(9):995-1002. 
8. Kappos L, Polman CH, Freedman MS, et al. Treatment with interferon beta- $1 \mathrm{~b}$ delays conversion to clinically definite and McDonald MS in patients with clinically isolated syndromes. Neurology. 2006;67(7):1242-9.

9. Brochet B, Lemaire G, Beddiaf A, et al. Reduction of injection site reactions in multiple sclerosis (MS) patients newly started on interferon beta $1 \mathrm{~b}$ therapy with two different devices. Rev Neurol (Paris). 2006;162(6-7):735-40.

10. Pozzilli C, Schweikert B, Ecari U, et al. Supportive strategies to improve adherence to IFN beta- $1 \mathrm{~b}$ in multiple sclerosis-results of the BetaPlus observational cohort study. J Neurol Sci. 2011;307:120-6.

11. Bayas A. Improving adherence to injectable disease-modifying drugs in multiple sclerosis. Expert Opin Drg Deliv. 2013;10(3):285-7.

12. Brennan PF, Strombom I. Improving health care by understanding patient preferences: the role of computer technology. J Am Med Inform Assoc. 1998;5(3):257-62.

13. Weller I, Saake A, Schreiner T, Vogelreuter J, Petroff N. Patient satisfaction with the BETACONNECT ${ }^{\mathrm{TM}}$ autoinjector for interferon beta-1b. Patient Prefer Adherence. 2015;9:951-9.

14. Ziemssen $\mathrm{T}$, Sylvester L, Rametta M, Ross AP. Patient satisfaction with the new interferon beta-1b autoinjector (BETACONNECT ${ }^{\mathrm{TM}}$ ). Neurol Ther. 2015;4:125-36.

15. di Cantogno EV, Russell S, Snow T. Understanding and meeting injection device needs in multiple sclerosis: a survey of patient attitudes and practices. Patient Prefer Adherence. 2011;5:173-80.

16. Bayas A, Japp G, Fulda U, Kallmann BA. Injection devices in MS therapy: survey on neurologists, MS-nurses and patients. Nervenheilkunde. 2010;29:57-62.
17. Treadaway K, Cutter G, Salter A, et al. Factors that influence adherence with disease-modifying therapy in MS. J Neurol. 2009;256(4):568-76.

18. Devonshire V, Lapierre $\mathrm{Y}$, Macdonell $\mathrm{R}$, et al. The global adherence project (GAP): a multicenter observational study on adherence to disease-modifying therapies in patients with relapsing-remitting multiple sclerosis. Eur J Neurol. 2011;18(1):69-77.

19. IMS Health. National prescription audits. January to December 2015.

20. Singer B, Wray S, Miller T, et al. Patient-rated ease of use and functional reliability of an electronic autoinjector for self-injection of subcutaneous interferon beta-1a for relapsing multiple sclerosis. Mult Scler Relat Disord. 2012;1(2):87-94.

21. Bayas A, Ouallet JC, Kallmann B, et al. Adherence to, and effectiveness of, subcutaneous interferon $\beta$-1a administered by RebiSmart ${ }^{\circledR}$ in patients with relapsing multiple sclerosis: results of the 1-year, observational SMART study. Expert Opin Drug Deliv. 2015;12(8):1239-50.

22. Baum K. Improving patient satisfaction with injection devices in multiple sclerosis improves adherence. Eur Neurol Rev. 2009;4(2):64-8.

23. Teva Pharmaceutical Industries Ltd. Capoxone ${ }^{\circledR}$. Taking Teva's Capoxone ${ }^{\circledR}$. https://www.copaxone. com/injection-assistance. Accessed 26 May 2016.

24. Novartis AG. Extavia ${ }^{\circledR}$ (interferon beta-1b). Dosage and Administration. https://www.extaviahcp.com/ administration. Accessed 26 May 2016.

25. EMD Serono, Inc. Rebif ${ }^{\circledR}$ (interferon beta-1a). Taking Rebif. http://www.rebif.com/taking-rebif/ rebif-administration-options. Accessed 26 May 2016. 